(Aus dem pflanzenphysiologischen Institut in Göttingen.)

\title{
Kreisen galvanische Ströme in lebenden Pfianzenzellen?
}

Von

\section{J. Teinte.}

"L'observateur qui est témoin pour la première fois du mouvement des globules de le lymphe dans le chara est porté à l'attribuer à l'électricité!" Mit diesen Worten beginnt Becquerel1) eine Abhandlung, welche sich auf die Frage bezieht, ob die Rotation der Protoplasmakörnchen in den Zellen der Characeen durch strömende Electricität hervorgebracht werde. In der That ist die an der einen Seite der Zelle anfsteigende, an der anderen Seite absteigende Bewegung eine solche, dass man wegen des analogen Verhaltens eines geschlossenen galvanischen Stromes zur Aufwerfung der von Becquerel gestellten Frage wohl berechtigt ist. A'uch darin muss man Becqu erel beipflichten, dass unsere Kenntnisse in der Electricitätslehre ausreichend sind, um diese Frage zu entscheiden.

Dennoch scheint mir der Weg, den Becquerel hierbei einschlug, nicht gerade der geeignetste zu sein. Derselbe brachte eine Charazelle unter ein Mikroskop, welches die Protoplasmaströmung erkennen liess, und führte eine Spirale, vermuthlich ans Kupferdraht, um die Zelle herum; die. Windungen der Spirale waren der Strömungsrichtung parallel. Als nun durch die Spirale ein starker Strom gesandt wurde und es nicht gelang, die Ge-

1) Influence de l'électricité sur la circulation du Chara. - Comptes rendus 1837 , S. 784 ff. 
schwindigkeit der Körnchen dadurch zu steigern oder zu vermindern, auch nicht, wenn die Richtung der Spirale gegen die Zelle in beliebiger Weise abgeändert wurde, so zog Becquerel daraus den Schluss, dass das Phänomen der Strömung nicht durch Electricität hervorgerufen werde.

Obgleich Becquerel sich nicht näher darüber ausspricht, so war seine Absicht bei dem eingeschlagenen Verfahren doch wohl diese, beim Oeffnen und Schliessen seines Stromes in der Charazelle einen Inductionsstrom zu erzeugen, welcher, der Protoplasmaströmung entweder gleich oder entgegengesetzt gerichtet, dadurch eventuell den Körnchen eine positive oder negative Beschleunigung ertheilen sollte. Immerhin ist dies Verfahren nicht einwurfsfrei, schon weil der Weg, welchen der inducirte Strom in der als geschlossener Leiter fungirenden Zelle einschlägt, sich nicht voraussehen lässt, und darum erscheint mir der Becquerel'sche Versuch nicht ausreichend zur Entscheidung der Frage, ob die Protoplasmakörnchen durch einen in der lebenden Zelle kreisenden galvanischen Strom in Bewegung gesetzt werden.

Neuerdings ist wieder durch Velten ${ }^{1}$ ) die gleiche Frage in Anregung gebracht worden, und formulirt derselbe sogar die Hypothese, dass: „die Ursache der Protoplasmabewegung in electrischen Strömen, die der lebende Zellinhalt selbst erzeugt, zu suchen sei". Diese Hypothese wird aber keineswegs durch die von Velten beobachteten Einwirkungen galvaniseher Ströme auf lebende Zellen gestiutzt, sondern nur der Umstand, dass es Velten gelang, durch starke Inductionsströme die in abgestorbenen Zellen befindlichen Körnchen in Rotation zu versetzen, scheint ihn zur Aufstellụng derselben bewogen zu haben. Die äusserliche Uebereinstimmung zwischen der Körnchenströmung des Protoplasma und der Fortführung materieller Theilchen, die in Wasser gelöst oder suspendirt sind, durch einen electrischen Strom, ist doch wohl zur Begrïndung solcher Hypothese noch nicht ausreichend. Die Möglichkeit, ich möchte sagen, der Verdacht, dass galvanische Ströme im

1) Velten, Einwirkung strömender Electricität auf die Bewegung des Protoplasmas, auf den lebendigen und todten Zellinhalt, sowie auf materielle Theilchen überhaupt. I. Einfluss des galvanischen Stroms auf das Protoplasma und dessen Bewegungen. II. Einfluss des galvanischen Stromes auf den todten Zellinhalt. Sitzber. der Akad. in Wien 1876. April u. October. 
Innern der lebenden Pflanzenzellen cireuliren, wird aber immerhin durch derartige Erwägungen rege erbalten.

Die endgültige Entscheidung dieser Frage ist von der grössten Wichtigkeit für die Physiologie des Stoffíechsels. Wenn man dariber nachdenkt, durch welche Kräfte der Anstoss zu den zahlreichen Stoffwechselbewegungen in der Zelle gegeben wird, gelangt man nothwendig zu dem Ergebniss, dass, wenn im Protoplasma galvanische Ströme kreisen, dieselben durch die von ihnen hervorgerufenen electrochemischen Wirkungen von einschneidendster Bedeutung für den Stoffumsatz sein müssen. Insofern wïrden wir dann wahrscheinlich hier einem Reciprocitäts-Phänomen gegenuiberstehen, als die electrischen Ströme, selbst unzweifelhaft ein Erzeugniss chemischer Spannungen, ihrerseits wieder eigenartige chemische Umsetzungen würden herbeiführen müssen.

Diese ernährungsphysiologische Seite der Frage erschien mir so wichtig, dass mir Hypothesen über die Ursachen der Zersetzungsprocesse im lebenden Protoplasma so lange gänzlich in der Luft zu schweben schienen, als nicht die immerhin mögliche und plausible Hypothese Velten's zur Gewissheit erhoben oder widerlegt worden war. Ich glaube, im Folgenden zeigen zu können, dass es mir gelungen ist, einen Beweis für die Nichtexistenz der Velten'schen Ströme zu erbringen.

Die Entscheidung war abhängig von der Zuverlässigkeit der anzuwendenden Beobachtungsmethode.

Wo man es bisher unternommen hat, den Körper lebender Thiere und Pflanzen auf das Vorhandensein galvanischer Ströme zu prüfen, da benutzte man als Erkennungsmittel die Gesetze der ponderomotorischen Fernewirkung strömender Electricität. Ein paar Electroden werden mit der Oberfläche des zu untersuchenden Körpers in Verbindung gesetzt, zwisehen ihnen ist ein Multiplicatordraht eingeschaltet, der in tuberaus zahlreichen Windungen einen leicht beweglich aufgehängten Magnet umläuft, dessen Ablenkung durch ein mit Spiegelablesung verbundenes Fernrohr beobachtet wird. Wird der Körper, den wir mit den Electroden betasten, wirklich von einem Strom durchflossen, so werden in unser Galvanometer immer nur Zweigströme von relativ geringer Intensität eintreten; durch die grosse Empfindlichkeit des Apparates ist jedoch dafür gesorgt, dass dieselben unserer Wahrnebmung nicht leicht entgehen können. Andererseits kann aber aus dem Umstande, dass 
wir eine Ablenkung des Magnets beobachten, keineswegs unbeding t darauf geschlossen werden, dass in dem lebenden Organismus, von dem wir ableiten, wirklich ein galvaniseher Strom existirt.

Wir erhalten z. B. auch eine Ablenkung, wenn wir die beiden erwärmten Pole eines Turmalins mit den beiden Enden des Multiplicatordrahtes verbinden; und doch wird dadurch nur die im Turmalin bestehende electrostatische Spannung angezeigt, keineswegs ein im Krystall vorhandener electrischer Strom. Wo wir also bei der Ableitung von einem Organismus einen Ausschlag am Galvanometer wahrnehmen, können wir mit völliger Sicherheit nur den Schluss ziehen, dass zwischen den Berïhrungspunkten der Electroden mit dem Körper eine electrische Spannung irgend welcher Art besteht. Dieselbe kann bedingt werden durch einen im Körper präexistirenden Strom, von welchem ein Zweig in den Multiplicatordraht eintritt, dass diese Folgerung aber keine nothwendige ist, zeigt das Beispiel des Turmalins. Neben diesem allgemeinen kommt aber noch ein für die Organismen specielles Bedenken in Betracht. Die lebenden Zellen und Gewebe gehören zu der grossen Classe der feuchten Leiter. Die electromotorischen, oder noch allgemeiner gesagt, die electrischen Eigenschaften der feuchten Leiter sind aber noch so gut wie gar nicht physikalisch studirt, and ehe dies nicht geschehen ist, muss es gewagt erscheinen, aus gewissen, von lebenden Organismen $a b$ geleiteten electromotorischen Wirkungen auf Ströme in ihrem Innern zu schliessen, welche mit dem Lebensprocess im Zusammenhange stehen sollen. Es sind zahlreiche Vorgänge denkbar, durch welche in beliebigen feuchten Leitern, welche der Luft ausgesetzt simd, electrische Spannungen erzeugt werden können, z. B. durch Verdampfungen, locale Quellungen, capillare Strömungen u. s. w. Und wenn an einem lebenden Gewebe sich electrische Erscheinungen zu erkennen geben, welche an dem gleichen Gewebe nach dessen Abtödtung verschwinden, so ist darum noch nicht ausgeschlossen, dass wir es hier nur mit der electromotorischen Function eines feuchten Leiters $\mathrm{zu}$ thun haben, ohne dass der Lebensprocess dabei irgendwie betheiligt gewesen wäre; denn es ist z. B. vorstellbar, dass zwischen verschiedenen Theilen eines anorganischen fenchten Leiters, z. B. in einem mit verschiedener Gartenerde gefüllten Blumentopf, electrische Spannungen bestehen, welche durch eine gleichförmige Temperaturerhöhung der ganzen Masse, 
wie sie z. B. durch Eintauchen in heisses Wasser erzielt wird, sich beseitigen lassen.

Diese Erwägungen liessen es mir anthunlich erscheinen, überhaupt den Versuch zu machen, Thonstiefel-Electroden von soleher Feinheit zu construiren, dass man damit von der Oberfläche einer grösseren Nitella-Zelle würde ableiten können. Denn selbst wenn dies gelingen, und wenn ein deutlicher Ausschlag am Galvanometer erfolgen sollte, wirde ich daraus doch nicht den Schluss zu ziehen wagen, dass die Nitella-Zelle von einem geschlossenen Strom durchflossen werde. Es muss daher nothwendig eine andere Methode ersonnen werden, wenn die in Rede stehende Frage in einer unbedingt gulltigen Weise entschieden werden soll.

Wenn ein galvianischer Strom in der Nitella-Zelle cireulirt, so wird derselbe nur durch eine von ihm ausgehende electrodynamische Wirkung in die Ferne nachgewiesen werden können; hierdurch wird das Gebiet der Erscheinungen, aus dem die Methode zu wählen ist, begrenzt. Dem gleichen Gebiete gehört ja auch das bis jetzt uibliche galvanoskopische Verfahren an; und in der That gelangt man zu einer branchbaren Methode, wenn man dies Verfahren umkehrt.

Bekanntlich iibt nicht bloss ein von einem Strom durchflossener Leiter einen ponderomotorischen Einfluss auf einen beweglich aufgehängten Magneten aus, sondern auch ein fester Magnet wirkt ponderomotorisch auf einen beweglichen Leiter. Wenn man z. B. annimmt, dass ein um eine Korkkngel gelegter Metallreif von einem geschlossenen Strom durchlaufen, der Kork aber auf Wasser schwimmend gedacht wird, so kann man dem Metallreif und damit dem Kork durch den genäherten Pol eines Magneten jede beliebige Stellung und Drehung ertheilen. Theoretisch kann man sich dabei den Metallreif ersetzt denken durch einen kleinen Magnetstab, der als Axe des Metallreifs normal zur Ebene desselben steht; die Lage des Nordpols an diesem theoretischen Magneten ergiebt sich nach der Ampère'schen Regel aus der Richtung des Stroms im Reif. Würde man in unserer schwimmenden Korkkugel diesen Magneten etwa durch ein hindurchgestecktes Streichbolz markiren, dessen Kopf den Nordpol bedentete, so wïrde man den Kopf dieses Streichholzes durch Annäherung eines wirklichen Magneten in jede beliebige Stellung zu bringen vermögen.

Die Empfindlichkeit eines solchen Apparates, d. h. die Leich- 
tigkeit, mit welcher die Korkkugel durch den genäherten Pol eines Magneten bewegt werden kann, ist proportional der Stromstärke im Metallreif und der Stärke des Magneten; dagegen umgekehrt proportional der Grösse des Trägheitsmomentes der Korkkugel. Wird der Strom im Metallreif sehr schwach, so muss man einen sehr starken Magneten anwenden, um noch eine ponderomotorische Wirkung zu erzielen, auch hier aber existirt eine Grenze der Wirkung, indem auch der stärkste Magnet wirkungslos wird, sobald die Intensität des Stromes im Leiter im Verhältniss zum Trägheitsmoment desselben bei einer gegebenen Suspension unter ein wirksames Minimum herabsinkt. In diesem letzteren Falle wird man mit dieser Methode einen Strom nicht mehr nachweisen können; wenn wir aber von solchen tiberaus schwachen Strömen absehen, so besitzt die Methode einen hohen Grad von Empfindlichkeit und dürfte in dieser Hinsicht der älteren, bisher stets angewandten kaum nachstehen.

Diesem aus einer Korkkugel, einem Metallreif and einem Streichholz construirten Modell muss nun unsere lebende Charazelle bei Annäherung an den Pol eines Magneten entsprechen. Fliesst ein geschlossener Strom im Innern ihres Protoplasmaleibes, so muss die Zelle, beweglich suspendirt, bei einer Aenderung der Lage des Magneten auch ihre Lage verändern; bleibt die Zelle aber unbeweglicb, so ist kein Strom in ihrem Innern vorhanden.

Diese Erwägungen veranlassten mich zur Construction eines folgendermassen beschaffenen Galvanoskops.

Auf einem horizontalen Brett sind zwei verticale Bretter befestigt, welche in einem Ausschnitt einen grossen horizontalen Electromagneten tragen, derart, dass der Magnet beliebig in horizontaler Richtung verschoben werden kann. Der Electromagnet besteht ans einem hohlen, d. $h$. in seiner Längsaxe durchbohrten Eisenkern von $200 \mathrm{~mm}$ Länge und $40 \mathrm{~mm}$ Durchmesser; der Durchmesser des axilen Lumens beträgt $10 \mathrm{~mm}$.

Dies Lumen kann durch einen glattgedrehten und leicht darin verschiebbaren Eisenstab ausgefiillt werden, wodurch der sonst hohle cylindrische Eisenkern zu einer soliden Barre ergänzt wird.

Man kann diesen axilen Eisenstab nach der einen Seite herausziehen, und hier an seinern äussersten Ende besitzt derselbe eine horizontale, ebene Ausfeilung, die bis auf seine Axe hinabreicht, und welche das Auflegen einer kleinen Glasplatte gestattet. Der 
Eisenkern dieses Electromagneten ist fünfmal umwunden mit einem $3 \mathrm{~mm}$ dicken nmsponnenen Kupferdraht, die Mäehtigkeit der Drahtschicht beträgt $17 \mathrm{~mm}$; an den beiden Enden wird der Diaht durch eine an den Eisenkern gelöthete $2 \mathrm{~mm}$ dicke Messingplatte gehalten. Dem Magneten kann durch eine kleine, ans 4 Bunsen'schen Flaschenelementen bestehende Batterie ein sehr starker Magnetismus verliehen werden. Dem Hauptmagneten gegeniiber befindet sich ein in ähnlicher Weise, aber unbeweglich aufgestellter, zweiter, etwas kleinerer Electromagnet; derselbe besitzt einen soliden Eisenkern, der nur an der einen Seite in eine verjüngte Spitze ausgezogen ist, welche eine ähnliche flache Ausfeilung trägt, wie der Hauptmagnet. Die Spitzen der beiden Magnete können nun beliebig einander genähert werden; lässt man zwischen beiden Spitzen etwa $10 \mathrm{~mm}$ Abstand, so kann man auf beide ein grosses Deckglas legen, an dessen unterer Seite ein Wassertropfen hängt, in welchem das zu untersuchende Object sehwebend suspendirt ist. Das Object wird beobachtet durch ein dem Apparat genähertes Mikroskop, bei welchem der gewöhnliche Objecttisch entfernt und der Fuss zurückgedreht ist, und dessen Tubus an einem längeren Horizontalarm befestigt wurde. Man benutzt bei der in Rede stehenden Zusammenstellung ausschliesslich ein schwaches Objectivsystem mit weitem Focalabstand. Soll nun die Einwirkung der beiden combinirten Magnete auf ein Object beobachtet werden, so setzt man dieselben durch einen Commutator mit der Batterie in Verbindung, und zwar so, dass von den einander zugekehrten Spitzen der beiden Magnete die eine zum Nordpol, die andere zum Südpol wird. Ist der im Tropfen des Deckglases hängende Gegenstand ein von einem geschlossenen Strom durchflossener Leiter, so wird derselbe unter dem Einflusse des sehr kräftigen electromagnetischen Systems eine feste Gleichgewichtslage annehmen; sobald man nun den magnetisirenden Strom der Batterie durch den Commutator umkehrt, muss auch das im Tropfen befindliche Object eine Drehung erfahren; und ebenso muss eine Bewegung desselben eintreten, wenn man es in beliebiger Lage zwischen die beiden Magnetpole bringt und dann erst die Kette schliesst; Voraussetzung ist dabei nur, dass die Kraftlinien der Magnete nicht genau normal auf der Ebene des Stromes im Objecte stehen. Wenn man unter solchen Umständen an einer lebenden unverletzten Pflanzenzelle, welche im Tropfen des Deckglases suspendirt ist, durch 
Schliessen der Kette oder durch Umkehrung des Stromes keinerlei Bewegung hervorzubringen vermag, so darf man daraus den Schluss ziehen, dass ein ihren Protoplasmaleib durchlaufender Strom nicht existirt; denn selbst wenn die Stärke dieses Stromes so gering sein sollte, dass sie zur völligen Ueberwindung der zwischen Deckglas, Object und Tropfen herrschenden Capillarwiderstände nicht ausreichte, würde doch unzweifelhaft die Zelle bei Schliessung und Umkehrung des Stromes in Zuckungen versetzt werden, die sich durch das Mikroskop müssten wahrnehmen lassen. Nimmt man aber an, dass die lebhafte Protoplasmaströmung in einer Charazelle durch einen galvanischen Strom verursacht wird, so kann die Stärke dieses Stromes gar nicht unbedeutend sein, sie muss unzweifelhaft hinreichen, eine im Wasser suspendirte Zelle bei Einwirkung unseres sehr kräftigen electromagnetischen Systems zu drehen. -

Als geeignetes Beobachtungsobject dienten mir junge, unberindete, erst etwa $1 \mathrm{~mm}$ lange Spitzen von Seitenstrahlen einer Chara fragilis, welche im Aquarium gezogen war. Diese jungen Zellen wurden unter dem Präparirmikroskop möglichst kurz abgeschnitten und, nachdem ich mich von ihrer Intactheit und ihrer lebhaften Protoplasmaströmung auch nach der Amputation überzengt hatte, in der Spitze eines ziemlich grossen, auf der Unterseite eines Deckglases hängenden Wassertropfens suspendirt, das Deckglas dann mit seinen beiden Rändern auf die Spitzen der beiden Electromagnete gelegt, von denen die des grösseren hierbei möglichst verkürzt wurde, so dass das Deckglas ganz nahe an den Hauptkörper des Magneten hinangerückt werden konnte. Dann wurde das Mikroskop auf die Zelle eingestellt, dessen Vergrösserung noch eben die Strömung erkennen liess. Die Beweglichkeit der im Wassertropfen suspendirten Zelle war eine so grosse, dass die leiseste Erschuttterung des Tisches dieselbe vibriren liess, und bei der geringfügigsten Hebung oder Senkung des Deckglases zeigte sie eine Verschiebung.

Es wurde nun, während ich durch das Mikroskop sah, der das System magnetisirende Strom geschlossen: keine Bewegung der Zelle. Es wurde die Richtung des Stromes dureh den Commutator gewechselt: die Zelle blieb anch jetzt unbeweglich, anch nachdem sie in verschiedenster Weise gedreht war, um der Rotationsrichtung der Strömung eine andere Stellung zur Axe der 
Magnete zu geben. Zahlreiche andere Charazellen verhielten sich wie die zuerst untersuchte; junge Internodien von Nitella, sowie Brennhaare von Urtica lieferten ein gleiches negatives Resultat ${ }^{1}$ ).

Aus diesen Versuehen scheint mir unwiderleglich zu folgen, dass die Rotation des Protoplasma in den Zellen der Characeen nicht durch einen galvanischen Strom hervorgerufen sein kann.

Mit einer geeigneten Abänderung lässt sich die von mir benutzte Methode noch in anderer Weise verwenden, um die Frage zu beantworten, ob einzelne Theile des Protoplasmaleibes von einem galvanischen Strom durchflossen werden, welcher die Körnchen desselben in Bewegung setzt. $\mathrm{Zu}$ dem Ende habe ich meinen galvanoskopischen Apparat in der folgenden Weise gestaltet.

Auf einem genau horizontal zu stellenden gusseisernen Fusse wird der grössere Electromagnet vertical aufgestellt und der centrale Eisenkern herausgezogen, so dass der Eisenstab des Magneten nunmehr von einer axilen Bohrung von $10 \mathrm{~mm}$ im Lichten durchzogen wird. Diese Bohrung wird durch einen, unter dem gusseisernen Fusse angebrachten Planspiegel erhellt. Im oberen Ende der Bohrung des Magneten ist eine Condensorlinse eingesetzt, so dass, wenn man ein mikroskopisches Präparat über diesen Condensor legt, dasselbe hell genug erlenchtet wird, um eine Betrachtung bei etwa 300 facher Vergrösserung zu gestatten. Das zum Beobachten dienende Mikroskop wird zu diesem Zwecke auf einem ziemlich hohen hölzernen Dreifuss dicht an den vertical stehenden Magneten herangerückt und die Einstellung auf das Präparat in gewöhnlicher Weise vollzogen. Um das zu untersuchende Object dem Eisenkern möglichst zu nähern, benutzte ich grosse Deckgläser als Objectträger.

Bei der Construction dieses letzteren Apparates ging ich von der Erwägung aus, dass ein über dem Pol eines vertical stehenden Magnetstabes befindliches, horizontal laufendes Stromelement

1) Es kann vorkommen, dass bei der Schliessung des Stromes eine suspendirte Charazelle ein wenig bewegt oder gedreht wird; dies rührt aber nachweislich immer nur daher, dass der Apparat nicht ganz fest zusammengesetzt war und durch die Magnetisirung des Eisens ein Ruck entstand. In solchen Fällen zeigen auch kleine Holzspähnchen u. dgl., welche in dem Wassertropfen suspendirt sind, eine Bewegung. 
durch die Einwirkung des Magneten einen horizontal gerichteten Impuls erhält, welcher das Stromelement um eine der Axe des Magneten parallele Axe zu drehen sucht. Wenn man daher einen horizontal iiber den $\mathrm{Pol}$ des Magneten hinweglaufenden kurzen Leiter in seinem Schwerpunkt beweglich aufhängt und dann einen Strom dureh ihn hindurchsehickt, so beginnt der Leiter um eine verticale Axe zu rotiren. Es ist dies der bekannte electromagnetische Rotationsversuch.

Mit einem solchen Leiter lässt sich ein dünner, quer durch den Saftraum der Zelle verlaufender Protoplasmafaden der Tradescantia-Haarzelle vergleichen. Wird die in demselben sichtbare Körnchenströmung durch einen electrisehen Strom hervorgerufen, so muss sich eine ponderomotorische Einwirkung auf den Protoplasmafaden unter dem Mikroskope bemerklich machen, weil der die Körnchenbewegung hervorbringende galvanische Strom gar nicht sehr schwach sein kann, und weil der zur Anwendung kommende Electromagnet ein sehr starker ist. Allerdings ist ein durch den Saftraum verlaufender Protoplasmafaden keineswegs an sich beweglich suspendirt, sondern wir müssen ihn mit einem an beiden Enden festgeknïpften Seil oder Draht vergleichen. Ein in solcher Weise befestigter und vom Strom durchflossener Draht würde allerdings von einem Magneten nicht in Bewegung gesetzt werden können; anders aber muss sich ein plastisch-weicher Protoplasmafaden verhalten. Nehmen wir an, das Ende a eines solchen Fadens sei nach Norden, das andere Ende b nach Süden gekehrt, so sucht der Magnet den Faden um seinen Halbirungspunkt $\mathrm{e}$ za drehen, und dabei wird sich seine Einwirkung so gestalten, dass das Ende a e des Fadens einen von Osten nach Westen, das Ende b e des Fadens einen von Westen nach Osten gerichteten Impuls erhält. Die Einwirkung des Magneten wird also dahin zielen, unter Dehnung des aus plastisch-weicher Materie bestehenden Fadens demselben eine S-förmige Krümmung zu verleihen, und scheint es mir wahrscheinlich, dass der Einfluss einer solchen Kraft sich unter dem Mikroskop als eine wenn auch nur geringfügige Zerrung des Fadens müsste bemerklich machen. Ferner dürfte wohl als sicher angenommen werden, dass die Masse der im Faden sich fortschiebenden Körnchen von strömender Electricität erfüllt sei, welche die Richtung der sichtbaren Körnerbewegung theilt. In diesem Falle müsste jedes einzelne Körnchen 
durch den Magneten einen seitlich gerichteten Impuls erhalten, welcher voraussichtlich dahin wirken würde, die Geschwindigkeit desselben zu verlangsamen, und welcher sich bei Schliessung der Kette unter dem Mikroskop bemerklich machen müsste.

Es wurden nun Versuche angestellt mit Zellen aus den Staub- fadenhaaren von Tradescantia virginica und Commelina erassula, wozu vorweg bemerkt sein möge, dass sich beide Pflanzen ganz gleich verhielten. Nachdem ein geeignetes Präparat hergerichtet war, wurde ein recht dünner Protoplasmafaden, der nur ein Strombett zeigte, unmittelbar neben einen längeren Theilstrich des Ocular-Mikrometers, und zwar parallel demselben, eingestellt, darauf der Faden eine Zeitlang beobachtet, um zu sehen, welche Veränderungen seiner Lage etwa durch spontane Massenverschiebungen im Protoplasma hervorgerufen wiirden. Dann schloss ich während der Beobachtung die Kette: der Faden blieb ganz ungeändert in seiner Lage; die Körnchen flossen in derselben Weise weiter wie sorher, ohne sich zu drehen oder auch nur zu zucken, auch die Geschwindigkeit der Strömung wurde nicht alterirt. Ebensowenig vermochte eine längere Dauer der Einwirkung des Magneten irgend welche Veränderungen der Fäden hervorzurufen, welche man auf Rechnung des Magnetismus hätte setzen müssen. Die Versuche wurden sehr oft und mit verschiedenen Objecten wiederholt, der magnetisirende Strom durch den Commutator umgekehrt u. s. w., niemals zeigte sich auch nur die leiseste Einwirkung auf das Protoplasma. Ein gleiches negatives Ergebniss lieferte die Untersuchung der Brennhaare von Urtica und der Wurzelhaare von Trianea bogotensis, ebenso der Blattzellen von Vallisneria spiralis; gerade bei diesem letzten Object hätte die Einwirkung des Magneten auf die einzelnen strömenden Chlorophyllkörner eine evidente sein mïssen.

Endlich sei noch hervorgehoben, dass ich auch bei dieser zuletzt beschriebenen Aufstellung des Magneten denselben auf ganze, in einem Wassertropfen hängende Charazellen einwirken liess, ebenfalls ohne jeden Erfolg.

Ich glaube aus diesen übereinstimmenden, negativen Ergebnissen meiner Untersuchangen den Schluss ziehen zu dürfen, dass strömende Electricität die Ursache der Körnerbewegung im Protoplasma von Pflanzenzellen nicht ist. Es liesse sich gegen diesen Schluss nur ein einziger Einwand erheben, nämlich der, dass die 
galvanischen Ströme der Zellen zu schwach waren, um durch meine Methode nachgewiesen werden zu können. Ich bin diesem Einwande schon mehrfach entgegen getreten, und hebe hier nochmals hervor, dass ich diese Methode für eine äusserst empfindliche halte. Dafür bürgt mir in erster Linie die Stärke des angewandten Electromagneten. Derselbe müsste unbedingt im Stande sein, die in dem Wassertropfen suspendirten Zellen, wenn auch nur Ströme von minimaler Stärke in ihnen circulirten, zu bewegen. Um aber die bekannten Bewegungserscheinungen des Protoplasma hervorzurufen, würde eine ziemlich beträchtliche electromotorische Kraft erforderlich sein, weil die Substanz des Protoplasma jedenfalls einen bedeutenden Widerstand besitzt.

Ich habe auch zahlreiche isolirte Pflanzenzellen, in denen eine Protoplasmaströmung weniger deutlich oder gar nicht zu erkennen war, der Einwirkung des Magneten ausgesetzt, aber immer ohne Erfolg. Aus diesen Gründen bin ich zu der Ansicht gelangt, dass circulirende galvanische Ströme in lebenden Pflanzenzellen überhaupt nicht existiren, weil diese Zellen sonst die ponderomotorische Einwirkung eines kräftigen Electromagneten miissten erkennen lassen. Ich muss auch bekennen, dass, je länger ich darüber nachdenke, mir die Möglichkeit des Bestehens solcher Ströme in den Zellen immer schwerer vorstellbar wird. -

Auf die zahlreichen, in der Literatur vorhandenen Angaben iber electromotorische Erscheinungen an lebenden Pflanzentheilen soll hier nicht eingegangen werden. Ich bemerke noch, dass ich mit den Darlegungen von Kunkel in dessen vortrefflichem Aufsatze "Ueber electromotorische Wirkungen an unverletzten lebenden Pflanzentheilen " 1) vollkommen einverstanden bin, und bedaure nur, dass dieser Autor nicht auch Dionaea muscipula in den Kreis seiner Untersuchungen gezogen hat.

1) Arbeiten des bot. Instituts in Würzburg, II, S. 1. 